\title{
Alta parasitemia pelo Trypanosoma cruzi em paciente com lupus eritematoso sistêmico
}

\author{
Trypanosoma cruzi high parasitemia in patient \\ with systemic lupus erythematosus
}

\author{
Leopoldo Luiz dos Santos-Neto', Máira F. Polcheira ${ }^{2}$ Cleudson Castro ${ }^{3}$, Rodrigo Aires Corrêa Lima', \\ César Kozak Simaan ${ }^{1}$ e Francisco Aires Corrêa-Lima1
}

\begin{abstract}
Resumo É descrito um caso de doença de Chagas com alta parasitemia pelo Trypanosoma cruzi em paciente com lupus eritematoso sistêmico. O xénodiagnóstico foi útil na identificação da parasitemia e o benznidazol foi capaz de reduzir a alta e incomum parasitemia. Em indivíduos com doenças auto-imunes e immunossuprimidos, o benznidazol pode ser uma alternativa no controle da alta parasitemia por Trypanosoma cruzi.

Palavras-chaves: Lupus eritematoso sistêmico. Doença de Chagas. Xenodiagnóstico. Benznidazol. Alta parasitemia.
\end{abstract}

Abstract $A$ case of Trypanosoma cruzi high parasitemia in patient with systemic lupus erythematosus is reported. A xenodiagnostic test was useful in the identification of high and uncommon parasitemia, and Benznidazole was able to reduce the parasitemia. Benznidazole can be an alternative to control the Trypanosoma cruzi high parasitemia in people with autoimmune disease and immunosuppression.

Key-words: Systemic lupus erythematosus. Chagas' disease. Xenodiagnostic test. Benznidazole. High parasitemia.

Indivíduos com infecção chagásica na fase crônica podem apresentar reativação durante o período de imunossupressão celular. Pacientes com neoplasias, SIDA ou transplantados em uso de ciclosporina ${ }^{135678}$ podem apresentar reativação, tendo como manifestações encefalite ou abscesso cerebral $^{27}$. Nessas circunstâncias tem sido consenso o uso de medicação antitripanossomicida para evitar as complicações da reativação. Por outro lado, pacientes em uso crônico de corticosteróide podem apresentar parasitemia, mas o emprego de drogas antitripanosomicida ainda é motivo de controvérsias ${ }^{10}$.

Apresentamos relato de caso de uma paciente com lupus eritematoso sistêmico (LES) que apresentou importante parasitemia em vigência da imunossupressão com corticóide e ciclofosfamida.

\section{RELATO DE CASO}

Mulher de 33 anos, natural do município de Posse (GO) com diagnóstico de LES há 12 anos e 3 sorologias (Hemaglutinação indireta e Imunofluorescência indireta) positivas para doença de Chagas. As primeiras manifestações do LES foram cutâneas, que responderam ao uso de talidomida. Em 1992 apresentou poliartrite, polineurite e síndrome nefrótica. Tinha um FAN de 1:512, anti-DNA positivo, C3:
91,1 mg/dl; C4: 2,9mg/dl; eletroneuromiografia mostrou ausência de resposta à estimulação de nervo sural direito e esquerdo e sinais leves de desnervação no músculo tibial anterior, compatível com polineuropatia periférica. A biópsia renal revelou glomerulonefrite mesangial lúpica, classe II-B, da OMS. Foi medicada com prednisona $1 \mathrm{mg} / \mathrm{kg}$ e ciclofosfamida em forma de pulsoterapia mensal, $1 \mathrm{~g} / \mathrm{m}^{2}$ de superfície corporal.

1. Serviço de Reumatologia do Hospital Universitário de Brasília da Faculdade de Medicina da Universidade de Brasília, Brasília, DF. 2. Curso de Graduação de Medicina da Faculdade de Medicina da Universidade de Brasília, Brasília, DF. 3. Serviço de Doença Infecto-parasitária do Hospital Universitário de Brasília da Faculdade de Medicina da Universidade de Brasília, Brasília, DF.

Endereço para correspondência: Dr. Leopoldo Luiz dos Santos Neto. Serviço de Reumatologia do HUB/FM/UnB, Caixa Postal 04438, 70192-970 Brasília, DF. Tel: 5561 448-5229, Fax: 61 349-8089

e-mail: leoneto@uninet.com.br

Recebido para publicação em 12/8/2002

Aceito em 6/8/2003 
Em março de 1992, durante o $2^{\circ}$ mês da pulsoterapia realizou um xenodiagnóstico natural ${ }^{4}$, com 40 triatomíneos, que examinados individualmente 30 dias após, mostrou que $37(92,5 \%)$ foram positivos para T. cruzi . Nessa ocasião a ciclofosfamida foi substituída pelo metotrexate e cloroquina e foi iniciado benznidazol $(5 \mathrm{mg} / \mathrm{kg} / \mathrm{dia})$ por 60 dias.

Durante 5 anos, permaneceu assintomática do LES, usando hidroclorotiazida ( $25 \mathrm{mg} / \mathrm{dia})$ e propanolol (40mg 2 vezes ao dia) para controle da hipertensão arterial. Três xenodiagnósticos naturais realizados em julho e dezembro de 1992 e um em novembro de 1993 foram negativos. Durante esse período o exame cardiovascular foi normal, O ECG foi normal e o ecocardiograma mostrou leve espessamento de pericárdio.

No ano 2000, houve recidiva do LES, caracterizado por poliartrite e febre. Nessa ocasião, apresentava hemoglobina de $11,2 \mathrm{~g} / \mathrm{dl}$; leucometria de 4.000 (eosinófilos: 4; bastões: 5, segmentados: 66; linfócitos: 22; e monócitos: 3) VHS: $41 \mathrm{~mm} / 1^{\text {a }}$ hora; uréia: $63 \mathrm{mg} / \mathrm{dl}$; creatinina: $0,9 \mathrm{mg} / \mathrm{dl}$; proteinúria $935 \mathrm{mg} /$ 24h; clareamento de creatinina: $51 \mathrm{ml} / \mathrm{min} / 1,72 \mathrm{~m}^{2}$; EAS com albumina +++; piócitos 19/campo; hemácias 25/ campo; presença de cilindros hialinos e granulosos. Foram reintroduzidos metotrexate $10 \mathrm{mg} / \mathrm{semana}$, cloroquina $150 \mathrm{mg} /$ dia e prednisona $10 \mathrm{mg} / \mathrm{dia}$. Após 2 semanas, foi internada com cefaléia, vômitos, vigil, orientada, com rigidez de nuca e estrabismo convergente bilateral, mais à esquerda e fundo de olho normal. O liquor estava claro, com citometria de $160 \mathrm{cel} / \mathrm{mm}^{3}$ (26\% de neutrófilos; $74 \%$ de mononucleares); glicose de $44 \mathrm{mg} \%$. Pesquisa direta para bactérias, fungos e protozoários foi negativa. A cultura para germes piogêncios e micobactéria foi negativo. $O$ ECG foi sugestivo de aumento de câmaras (AE e VE). No segundo dia de internação foi a óbito. A necropsia mostrou meningoencefalite, edema agudo de pulmão e vasculite generalizada, secundária ao LES. A causa mortis foi edema agudo de pulmão. O exame histopatológico não identificou a presença de amastigotas no coração, cérebro, fígado e baço. Não havia infiltrado, fibrose, miocitólise ou denervação que sugerisse a presença de miocardiopatia chagásica. No entanto, foram identificadas lesões, agudas e crônicas, sugestivas de vasculite generalizada (Figuras $1 \mathrm{~A}$ e $\mathrm{B}$ ). O xenodiagnóstico artificial ${ }^{11}$ realizado durante a internação mostrou que os oitos pools examinados foram negativos.

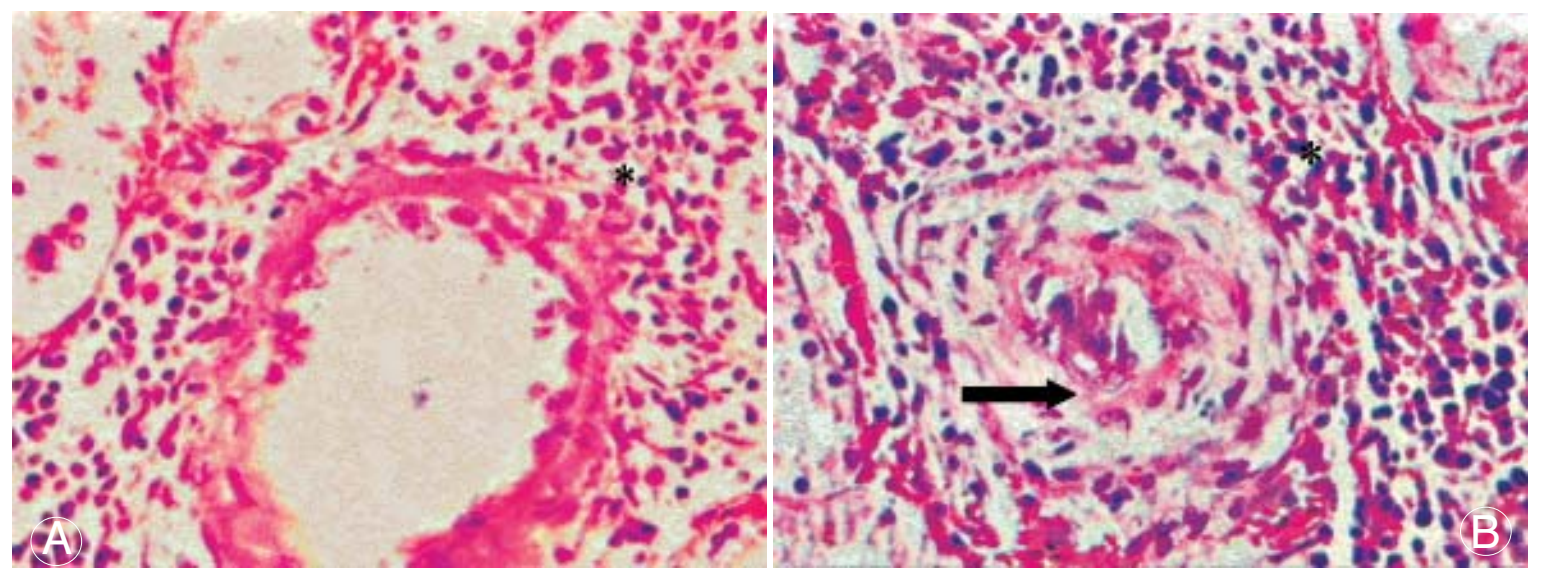

Figura 1 - Diferentes fases da vasculite. A- Fase aguda; B-Fase crônica: Necrose hialina da parede da arteríola renal (seta) e infiltrado mononuclear linfoplasmocitário vascular e perivascular (asterisco)(HE, $250 X)$.

\section{DISCUSSÃO}

A associação do LES com doença de Chagas constitui um dilema terapêutico, já que é um evento clínico pouco freqüente. Relatamos um caso de LES em atividade, que apresentava sorologia positiva para doença de Chagas. Durante o uso de ciclofosfamida e corticosteróide, foi detectada elevada parasitemia através do xenodiagnóstico, com tendência a reativação, pois $92,5 \%$ dos triatomíneos foram infectados. Optou-se pelo uso profilático do benznidazol, que foi eficaz no controle da parasitemia. Após 8 anos de acompanhamento, a paciente veio a falecer de vasculite lúpica generalizada. Nessa ocasião, não foram identificadas amastigotas em tecido cerebral, cardíaco ou hepático. O xenodiagnóstico também foi negativo, corroborando a negativação do parasitismo.

O controle da parasitemia, com o uso de drogas antitripanossomicidas, nessa situação clínica ainda não está definida. Os relatos de casos são inconclusivos. Barousse e cols ${ }^{2}$ relataram 10 pacientes chagásicas com LES que fizeram profilaxia com nifurtimox. Apenas cinco pacientes fizeram uso de ciclofosfamida e corticosteróide, sendo que três foram a óbito. Em nenhum dos casos necropsiados, foram identificadas amastigotas de $T$. cruzi. Todos os pacientes que permaneceram em acompanhamento não apresentaram reativação. No entanto, esses mesmos autores descreveram quatro casos de pacientes 
chagásicos que apresentavam tumores sólidos ou hematológicos, nos quais não foi realizada profilaxia antitripanossomicida. Em nenhum desses casos estudados, foi identificada reativação da doença de Chagas. A reativação da doença de Chagas foi descrita num paciente chagásico com Linfoma de Hodgkin que utilizou corticosteróide e ciclofosfamida ${ }^{1}$. Outro relato foi uma criança que apresentava a forma congênita e a reativação foi atribuída ao uso de dexametasona ${ }^{14}$.

A presença de parasitemia é um dado importante no paciente chagásico imunossuprimido, já que pode anteceder em $22,2 \%$ as manifestações clínicas associadas com a reativação da doença de Chagas $^{3}$. Porém, a presença de alta parasitemia nesse contexto é muito mais relevante. $O$ uso de medicação antitripanossomicida em pacientes com forma crônica, fase indeterminada ou portador de miocardiopatia, é outro assunto também controverso. A indicação profilática é mais estabelecida no uso profilático em situações clínicas associadas a grave imunossupressão celular, tais como pacientes transplantados e em uso de drogas imunossupressoras. No entanto, a profilaxia em pacientes chagásicos, que fazem uso crônico de corticosteróide, ainda está sujeita a controvérsias, tendo alguns autores indicado benefícios na profilaxia primária ${ }^{12}$, enquanto outros apontam para a falta de estudos clínicos controlados para embasar essa indicação ${ }^{9}$. Nos casos relatados de reativação da doença de Chagas, existe uma evidente redução da resposta imune celular, tais como SIDA $^{5}$, transplantados ${ }^{3}$ e neoplasias malignas ${ }^{9}$.

O nosso caso trata-se de paciente lúpica, com um grau de imunossupressão mais intenso, devido à associação de corticosteróide e ciclofosfamida. Foi identificada parasitemia incomum, durante o período do uso desses dois medicamentos, semelhante aos casos descritos por Rassi e Amato $^{13}$ em pacientes que usavam só corticosteróide. Esses autores ${ }^{12}$ mostraram que o uso de $10 \mathrm{mg} / \mathrm{kg}$, por 60 dias apresentou uma redução significativa da parasitemia nos pacientes chagásicos. A dose empregada de benznidazol em nossa paciente foi a preconizada pelo Ministério da Saúde de $5 \mathrm{mg} / \mathrm{kg}$ de peso. Essa dose foi suficiente para suprimir a parasitemia, não tendo sido identificado parasito, nem no xenodiagnóstico, nem através do exame histopatológico.

O xenodiagnóstico muito positivo pode anteceder o aparecimento das manifestações clínicas da reativação da doença de Chagas em até $22,2 \%$ dos casos $^{3}$. Nesse caso, é aconselhável o uso de medicação antitripanossomicida na profilaxia de indivíduos chagásicos com doenças auto-imunes, em fase de imunossupressão. O emprego periódico do xenodiagnóstico artificial, assim como a profilaxia da reativação deve ser confirmada em estudos clínicos controlados.

\section{REFERÊNCIAS BIBLIOGRÁFICAS}

1. Almeida HO, Tafuri WL, Bogliolo L, Cunha JC. Parasitismo incomum do miocárdio e do esôfago em chagásico crônico, portador de Doença de Hodgkin e em uso de imunossupressores. Revista da Sociedade Brasileira de Medicina Tropical 8:117-121, 1974.

2. Barousse AP, Costa JA, Eposto M, Laplume H, Segura EL. Chagas disease and immunosuppression. Medicina (Buenos Aires) 40 (suppl 1):17-26, 1980

3. Bocchi EA. Transplante cardíaco em portadores de cardiopatia chagásica. Revista da Sociedade de Cardiologia do Estado de São Paulo 4:198-204, 1994.

4. Brumpt E. O xenodiagnóstico. Aplicação ao diagnóstico de algumas infecções parasitária e em particular a Trypanosomose de Chagas. Annaes Paulista de Medicina e Cirurgia 3:97-102, 1914.

5. Chocair PR, Amato Neto V, Sabaga E, Torrecillas PH. Aspectos clínico-diagnósticos relativos à fase aguda da doença de Chagas, em pacientes submetidos à transplante de rim e imunodeprimidos. Revista da Sociedade Brasileira de Medicina Tropical 18:43-45, 1985

6. Del Castillo M, Mendoza G, Oviedo J, Perez Bianco RP, Anselmo AE, Silva M. AIDS and Chagas' disease with central nervous system tumor-like lesion. American Journal and Medicine 88:693-694, 1990.

7. França LCM, Fleury RN Ramos Junior HA, Lemos S, Melaragno Filho R, Pasternak J. Moléstia de Chagas crônica associada a leucemia linfática: ocorrência de encefalite aguda como alteração do estado imunitário. Arquivos de Neuro-Psiquiatria 27:59-66, 1969.
8. Kohl S, Pickering LK, Frankel LS, Yaeger RG. Reactivation of Chagas' disease during therapy of acute lymphocytic leukemia. Cancer 50:827-828, 1982.

9. Lopez-Blanco OA, Cavalli NH, Jasovich A, Gonzalez-Cappa S, Nadal MA, Boschi A, Argüello EA, Stamboulian D, Favaloro R, Gotlieb D. Kidney transplantation and Chagas' disease. A twoyear follow-up of a patient with parasitemia. Transplantation 36:211-213, 1983.

10. Nishioka, SA. Benznidazol na quimioprofilaxia primária da reativação de doença de Chagas em chagásicos crônicos em uso de corticosteróides em doses imunodepressoras: há evidência suficiente para a recomendação do seu uso? Revista da Sociedade Brasileira de Medicina Tropical 33:83-85, 2000.

11. Nussenzweig V, Sontag R. Xenodiagnostico artificial. Novo processo. $1^{\text {os }}$ resultados positivos. Revista Paulista de Medicina 40:69-71, 1952

12. Rassi A, Amato-Neto V, Siqueira AF, Ferriolli Filho F, AmatoVS, Rassi Junior A. Benznidazol as a prophylactic drug to prevent reactivation in chronic chagasic patients treated with corticoid for associated diseases. Revista da Sociedade Brasileira de Medicina Tropical 32:475-482, 1999

13. Rassi A, Amato Neto V, de Siqueira AF, Doles J, Leite MS, Silva $\mathrm{OQ}$, Cardoso VM. The influence of corticoids, in Chronic Chagas disease, administered in virtue of associated disorders. Revista da Sociedade Brasileira de Medicina Tropical 30:293-299, 1997.

14. Tresoldi AT, Belangero VMS, Silva PEMR, Netto AA, Marques MC, Mezzacappa MAMS, Altemani AMAM. Doença de Chagas Congênita: Reagudização pela corticoterapia. Pediatria (São Paulo) 6:207-210, 1984. 